\title{
Una aproximación a la problemática del bullying en deportistas escolares y juveniles cubanos
}

\author{
An approach to the problematic of bullying in cuban school \\ and youth athletes
}

Roxana Toledo Vidal', Tania Tintorer Silva² \& Osvaldo Hernández González ${ }^{3}$

Toledo, R., Tintorer, T., \& Hernández, O. (2021). Una aproximación a la problemática del bullying en deportistas escolares y juveniles cubanos. Revista Ciencias de la Actividad Física UCM, No22(1), enero-junio, 1-8. DOI: http://doi.org/10.29035/rcaf.22.1.8

\section{RESUMEN}

El objetivo del presente estudio es describir los roles del bullying en el deporte en una Escuela de Iniciación Deportiva Escolar (EIDE) cubana y compararlos por género, categorías escolares y deportes. Estudio descriptivo, transversal, de una muestra no probabilística por conveniencia, de 173 deportistas (42,2\% mujeres y 57,8\% hombres) cubanos con edades entre 8 y 18 años hasta el momento de realizar el diagnóstico. Se evaluó la incidencia del bullying mediante un cuestionario que incluía 70 ítems. Los resultados revelan que no hubo diferencias significativas respecto al género, las categorías y los deportes estudiados. No obstante, se observó que los deportistas (M) mostraron valores más elevados en el rol de víctima y agresor, mientas que las deportistas (F) lo hicieron en el rol de observador. Asimismo, los escolares presentaron valores promedios más elevados en el rol de agresor y observador con respecto a los juveniles. La percepción de la incidencia del bullying en los deportes variaron en el rol de la víctima, el agresor y el observador. Los resultados sugieren que los episodios de bullying constituyen un problema clave en las escuelas deportivas cubanas que merece una mayor atención psicopedagógica, para que los deportistas puedan desempeñarse con éxito.

Palabras clave: Bullying, deportistas, deporte y ejercicio.

\section{ABSTRACT}

The objective of this study is to describe the roles of bullying in sports in a Cuban School for School Sports Initiation (EIDE) and to compare by gender, school categories and sports. Descriptive, cross-sectional study of a nonprobabilistic convenience sample of 173 Cuban athletes (42.2\% women and 57.8\% men) aged between 8 and 18 years up to the time of evaluation. The incidence of bullying was evaluated using a questionnaire that included 70 items. The results show that there were no significant differences regarding gender, categories and sports studied. However, it was observed that the athletes (M) showed higher values in the role of victim and aggressor, while the athletes (F) did so in the role of observer. Likewise, schoolchildren showed higher average values in the role of aggressor and observer with respect to juveniles. The perception of the incidence of bullying in sports varied in the role of the victim, the aggressor and the observer. The results suggest that bullying episodes constitute a key problem in Cuban sports schools that deserve greater psychopedagogical attention, so that athletes can perform successfully.

Key words: bullying, athletes, sport and exercise.

\footnotetext{
${ }^{1}$ Centro Provincial de Medicina del Deporte, Matanzas, Cuba. https://orcid.org/0000-0002-4251-1680

${ }^{2}$ Centro Provincial de Medicina del Deporte, Matanzas, Cuba. https://orcid.org/0000-0003-4809-8644

${ }^{3}$ Universidad de Talca, Talca, Chile. https://orcid.org/0000-0002-1319-6167
} 


\section{INTRODUCCIÓN}

El bullying o acoso escolar ha sido conceptualizado como un tipo de violencia escolar de carácter intencional, repetitivo y en el que se da un desbalance de poder entre las partes involucradas (víctima, agresor, y observadores), de gran repercusión psicológica para las víctimas (Sanmartín, 2007). La prevalencia de episodios de bullying ha crecido exponencialmente y las consecuencias para el desarrollo de las personas involucradas lo han convertido en un problema de salud pública (Bullying sin Fronteras, 2019, Morcillo et al., 2014, Rech et al., 2013). Estudios recientes incluyen además de la víctima y el agresor, a los múltiples observadores que participan en la dinámica del bullying adoptando una actitud pasiva que valida el comportamiento en apoyo al agresor y no se solidariza con la víctima (Garaigordobil \& Martínez, 2018, Malamut et al., 2020). Esto problematiza aún más la naturaleza de los episodios conductuales.

La literatura subraya que las personas que experimentan episodios de bullying en las primeras edades del desarrollo, pueden presentar más adelante problemas psicopatológicos, conductas suicidas y criminalidad (Brunstein et al., 2015). Los problemas psicológicos, sociales y educativos derivados de los episodios de bullying constituyen una realidad preocupante en el escenario educacional, por las consecuencias a corto y largo plazo que pueden producirse obstaculizando, en proporciones variables, el desarrollo de la personalidad (Silva et al., 2017). En este sentido, un estudio realizado recientemente por Chai et al. (2020), puso de relieve que las víctimas de bullying tienen mayores probabilidades de tener malas relaciones con los padres, maestros y compañeros, lo cual influye en su evaluación de la salud mental y la satisfacción con la vida.

Sin embargo, si bien en la literatura existe un consenso sobre cómo los episodios de bullying obstaculizan el desarrollo cognitivo, emocional y conductual de los niños, niñas y adolescentes, en las escuelas deportivas la comprensión y alcance del bullying continúa demandando atención (Bachand, 2017). Los estudios del bullying en el deporte muestran que su naturaleza competitiva tiende a promover e incrementar la agresión entre pares. De acuerdo a los criterios de Evans et al. (2016), en los atletas masculinos con entrenadores masculinos la prevalencia del bullying es aún mayor, debido a que los entrenadores minimizan o devalúan esta conducta entre los deportistas por considerarla como burlas comunes. Estos episodios de acoso impulsan interacciones negativas y empobrecen el clima deportivo

En este sentido, Stirling et al. (2011) han puesto de relieve que a las víctimas de bullying en los espacios deportivos les cuesta más trabajo romper el silencio y hablar de su experiencia de acoso, pues esta conducta se consideraría una debilidad mayor en este contexto. Sin lugar a dudas, la comunicación de episodios de bullying puede ayudar a romper el círculo de agresión, y promover un sentimiento de seguridad en las víctimas (Smokowski \& Evans, 2019). Es primordial que éstas cuenten con el apoyo necesario y oportuno, puesto que les pudiera ayudar a desarrollar estrategias que contribuyan a la solución del problema, en lugar de apostar por aquellas centradas en la desregulación emocional que perpetúen las experiencias ligadas a la experiencia de acoso (Nery et al., 2018)

En Cuba, el bullying ha sido poco estudiado, pero según Ruiz (2018) constituye un problema que ha venido incrementándose en los espacios escolares durante los últimos lustros. Hasta donde sabemos, son muy escasos los estudios que investigan la problemática del bullying en escuelas deportivas (Adler, 2014), a pesar de que la literatura sostiene que durante las prácticas deportivas las situaciones de acoso pueden ser más habituales y, por lo mismo, naturalizadas. En el presente trabajo se planteó como objetivo de investigación describir los roles del bullying en el deporte en una Escuela de Iniciación Deportiva Escolar (EIDE) cubana y compararlos por género, categorías escolares y deportes. Este estudio puede contribuir a poner de relieve el estado actual de la problemática del bullying en las escuelas deportivas y sus resultados podrían servir de base para la organización y el desarrollo de prácticas 
educativas preventivas, que contribuyan a ponerle barreras al problema, haciendo de la escuela un entorno más agradable y seguro.

\section{MÉTODOS}

\section{Tipo de estudio y muestra}

El presente estudio se realizó sobre la base de un diseño no experimental, transeccional y de alcance descriptivo-comparativo. Se estudiaron 173 deportistas (42,2\% mujeres y 57,8\% hombres) de una EIDE cubana. El rango de edad osciló entre 8 y 18 años a la fecha de la evaluación. La selección de la muestra fue de tipo no-probabilística (por conveniencia). Los participantes pertenecían a 11 de los deportes que se practican en la EIDE: natación, natación artística, clavados, esgrima, baloncesto, voleibol, hockey sobre césped, fútbol, boxeo, ciclismo y levantamiento de pesas. El 58,4\% de los participantes eran de la categoría escolar y el $41,6 \%$ de la juvenil.

Se incluyó en el estudio a los deportistas que pertenecían a la EIDE objeto de la investigación y que autorizaron libre y voluntariamente la carta del consentimiento informado. Se excluyó a los que se ausentaron durante los días de la aplicación del cuestionario. El estudio contó con la aprobación de los directores de la EIDE y del Centro Provincial de Medicina del Deporte de Matanzas, respectivamente. Todo el proceso de recolección de datos estuvo de acuerdo a las normas establecidas por la Declaración de Helsinki.

\section{Procedimientos}

La recolección de los datos se realizó durante los meses de septiembre y noviembre de 2019. La aplicación del cuestionario se llevó a cabo en horarios de clases y entrenamiento, en un aula dentro de la escuela que permitió conversar tranquilamente y con privacidad. La recogida de datos estuvo a cargo de los investigadores responsables del estudio. La exploración del bullying se efectuó mediante la técnica de la encuesta, aplicándose un cuestionario con 70 ítems (en escala tipo Likert de cinco valores numéricos del 1 al 5, donde el 1 es nunca, 2 es casi nunca, 3 es con frecuencia, 4 es casi siempre y 5 es siempre) de medición propuesto por Estrada \& Jaik (2011). Los deportistas contaron aproximadamente con un periodo de tiempo de 20 a 35 minutos para responder los ítems del cuestionario por medio el método tradicional de lápiz y papel.

Durante la aplicación del cuestionario uno de los investigadores responsables del estudio permaneció en el aula para despejar cualquier duda que tuvieran los participantes acerca del mismo. Para determinar la validez de contenido del instrumento, mediante el método de Lawshe, se utilizó el juicio de expertos (los 7 expertos provenían del campo de la psicología clínica y educacional con experiencia en la problemática del bullying). Los expertos evaluaron los reactivos del cuestionario de acuerdo a las siguientes categorías: rigor científico en los instrumentos elaborados; secuencia adecuada en los ítems a responder; correspondencia entre el objetivo del método y el instrumento; claridad del ítem a responder en el instrumento y grado de relevancia de la información que se obtiene. La apreciación valorativa de los expertos (0.78) se calculó por medio de la siguiente fórmula:

$$
C V R=\frac{n_{e}-(N / 2)}{N / 2}
$$

donde: $n_{e}=$ número de panelistas que tienen acuerdo en la categoría "esencial" y $N$ = número total de panelistas. La confiabilidad del instrumento se comprobó mediante el coeficiente de alfa de Cronbach, el cual mostró un valor de ( $r=0.71)$.

\section{Análisis estadístico}

En el estudio se utilizó la estadística descriptiva para determinar frecuencia (fi), porcentaje (\%), promedio (X) y desviación estándar (DE). Se verificó la normalidad de los datos de la encuesta por medio de la prueba de Kolmogorov-Smirnov. Las diferencias significativas según el género, la categoría y los deportes de los participantes se verificaron por medio de la prueba t de Student para muestras independientes y Anova de un factor. La probabilidad de margen de error adoptada fue de $\mathrm{p}<0.05$ y el tratamiento de 
los datos se efectuó por medio de las bondades operativas del programa estadístico SPSS $(22,0)$.

\section{RESULTADOS}

En la Tabla 1 se observan los valores medios y desviación estándar de la variable bullying desde la perspectiva de la víctima, del agresor y el observador de acuerdo al género de los participantes. No se verificó diferencias significativas $(p<0,005)$ respecto al género, a pesar de que los escolares masculinos presentaron valores promedios más elevados respecto al rol de víctima y agresor, mientras que los escolares del género femenino presentaron valores más elevados en el rol de observador.

Tabla 1

Valores medios y desviaciones estándar (DE) del bullying desde la perspectiva de víctima, agresor y observador de acuerdo a los deportes.

\begin{tabular}{lcccccc}
\hline \multirow{2}{*}{ Género } & \multicolumn{2}{c}{ Víctima } & \multicolumn{2}{c}{ Agresor } & \multicolumn{2}{c}{ Observador } \\
\cline { 2 - 7 } & $X$ & DE & $X$ & DE & $x$ & DE \\
\hline Femenino & 44,9 & 11,9 & 36,3 & 9,9 & 55,0 & 17,2 \\
Masculino & 46,6 & 14,8 & 43,1 & 17,0 & 53,8 & 21,0 \\
\hline
\end{tabular}

Leyenda: X: Promedio, DE: Desviación estándar.

En la Tabla 2 se muestran los valores medios y desviación estándar de la variable bullying desde la perspectiva de la víctima, del agresor y el observador de acuerdo a las categorías deportivas. No se verificó diferencias significativas $(p<0,005)$ respecto a las categorías, a pesar de que los escolares mostraron valores más elevados respecto al rol de agresor y observador con respecto a los juveniles.

Tabla 2

Valores medios y desviaciones estándar (DE) del bullying desde la perspectiva de víctima, agresor y observador de acuerdo a las categorías.

\begin{tabular}{lcccccc}
\hline \multirow{2}{*}{ Categorías } & \multicolumn{2}{c}{ Víctima } & \multicolumn{2}{c}{ Agresor } & \multicolumn{2}{c}{ Observador } \\
\cline { 2 - 7 } & $X$ & DE & $X$ & DE & $\times$ & DE \\
\hline Escolar & 45,8 & 13,0 & 42,1 & 15,9 & 56,2 & 19,4 \\
Juvenil & 46,3 & 13,7 & 38,1 & 12,3 & 51,6 & 18,8 \\
\hline
\end{tabular}

Leyenda: X: Promedio, DE: Desviación estándar.

En la Tabla 3 se observan los valores medios y desviación estándar de la variable bullying desde la perspectiva de la víctima, del agresor y el observador de acuerdo a los deportes evaluados. No se verificó diferencias significativas $(p<0,005)$ respecto a los deportes. Sin embargo, es posible notar que el promedio de víctimas en clavados, natación y baloncesto es superior a los demás deportes. En el rol de agresor el fútbol, el clavadosy el levantamiento de pesas presentaron promedios más elevados de bullying que el resto de los deportes. En cuanto al rol de observador la natación artística, esgrima, el voleibol, fútbol y levantamiento de pesas presentaron valores superiores a los deportes restantes. 
Tabla 3

Valores medios y desviaciones estándar (DE) del bullying desde la perspectiva víctima, agresor y observador de acuerdo a los deportes.

\begin{tabular}{lcccccc}
\hline \multirow{2}{*}{ Deportes } & \multicolumn{2}{c}{ Víctima } & \multicolumn{2}{c}{ Agresor } & \multicolumn{2}{c}{ Observador } \\
\cline { 2 - 7 } & X & DE & X & DE & X & DE \\
\hline Baloncesto & 46,3 & 11,8 & 34,9 & 6,9 & 39,3 & 12,9 \\
\hline Hockey (césped) & 37,8 & 8,41 & 31,1 & 3,7 & 34,6 & 13,8 \\
\hline Natación & 48,7 & 8,19 & 37,8 & 10,0 & 46,4 & 10,3 \\
\hline Voleibol & 45,5 & 8,86 & 43,1 & 12,0 & 56,3 & 12,0 \\
\hline Natación artística & 43,7 & 10,8 & 32,4 & 4,23 & 56,0 & 14,9 \\
\hline Fútbol & 45,5 & 10,2 & 52,3 & 19,5 & 68,1 & 21,9 \\
\hline Boxeo & 35,0 & 17,7 & 34,5 & 14,5 & 39,0 & 22,1 \\
\hline Ciclismo & 35,4 & 8,09 & 35,8 & 23,8 & 43,3 & 23,4 \\
\hline Lev. de Pesas & 40,4 & 10,9 & 51,6 & 19,1 & 71,0 & 18,2 \\
\hline Clavados & 57,2 & 9,6 & 46,0 & 10,3 & 68,9 & 12,6 \\
\hline Esgrima & 46,6 & 17,7 & 45,1 & 19,8 & 64,6 & 14,5 \\
\hline
\end{tabular}

Leyenda: X: Promedio, DE: Desviación estándar.

\section{DISCUSIÓN}

El objetivo de este estudio consistió en describir los roles del bullying en el deporte en una Escuela de Iniciación Deportiva Escolar (EIDE) cubana y compararlos por género, categorías escolares y deportes. Los resultados evidenciaron que no hubo diferencias significativas cuando se compararon los roles del bullying por género, las categorías (escolares y juveniles) y los deportes estudiados (natación, natación artística, clavados, esgrima, baloncesto, voleibol, hockey sobre césped, fútbol, boxeo, ciclismo y levantamiento de pesas). No obstante, se observó que los deportistas masculinos mostraron valores más elevados en el rol de víctima y agresor, mientas que las deportistas femeninas, lo hicieron en el rol de observador. Estos resultados son consistentes con otros estudios que han corroborado que los niños participan en calidad de víctima y agresor en más episodios de bullying que las niñas (Pepler et al., 2006). Sin embargo, existe evidencia que subraya que las personas del género femenino pueden verse más afectadas por los efectos depresivos de los episodios de bullying que los hombres (Holbrook et al., 2020).
Björkqvist (2018) asevera que las personas del género masculino son más propensas a conductas agresivas directas e indirectas en su modo de relacionarse con los demás, mientras que las personas del género femenino utilizan con más recurrencia prácticas de agresión indirecta. Esto puede explicar por qué los episodios de bullying en calidad de víctima y agresor son más habituales en los deportistas del género masculino. Es necesario subrayar que esta evidencia no es absoluta, puesto que la literatura revela la falta de unanimidad en los hallazgos relacionados con el género en los episodios de bullying y cyberbullying (Machimbarrena \& Garaigordobil, 2018). En este sentido, otros estudios han encontrado una ligera prevalencia del género femenino sobre el masculino en los distintos roles y acciones que componen los episodios del bullying en los escenarios educacionales (Domínguez et al.,2019). Esto indica la necesaria realización de estudios longitudinales que nos proporcionen un visión más global y holística de la dinámica del bullying.

Los resultados de la investigación mostraron, además, que en la percepción de los episodios de bullying, los deportistas escolares poseían valores 
promedios más elevados en el rol de agresor y observador con respecto a los deportistas juveniles. De acuerdo con los criterios de Vveinhardt \& Fominiene (2020), la edad de los deportistas constituye una variable clave en la aparición y duración de los episodios de bullying, ya que las personas con más experiencia de vida utilizan un arsenal comportamental negativo más elaborado, lo cual ayuda a invisibilizar los episodios de bullying. Sin embargo, en un estudio realizado por Ávila et al. (2010), los hallazgos indicaron que la edad de los participantes (estudiantes de 11 y 16 años, con una media de 13,76 años - DT = 1,815) no mostraba evidencias concluyentes (asociaciones significativas) en relación con las conductas del bullying. La diferencia entre estos resultados puede estar dada por la complejidad del fenómeno del bullying, en el que intervienen disímiles factores.

Los hallazgos del estudio también revelaron que la percepción de la incidencia del bullying en los deportes varió en el rol de la víctima, del agresor y del observador. En este sentido, el promedio de víctimas en clavados, natación y baloncesto fue superior a los demás deportes. En el rol de agresor el fútbol, clavados y levantamiento de pesas presentaron promedios más elevados de bullying que el resto de los deportes. En cuanto al rol de observador la natación artística, esgrima, voleibol, fútbol y levantamiento de pesas mostraron valores superiores a los deportes restantes. En la revisión de investigaciones especializadas en la problemática del bullying en el contexto deportivo, no se han encontrado análisis de diferencias significativas entre atletas de varios grupos de deportes, lo cual indica que se debe profundizar en este sentido.

En consecuencia, es necesario subrayar que este estudio tiene algunas limitaciones que deben ser mencionadas, por ejemplo, el método de muestreo utilizado es no-probabilístico, lo cual impide generalizar los resultados de la investigación. No se utilizaron variables, las que también pueden contribuir a explicar las conductas de bullying como la condición socioeconómica, el lugar de procedencia de los deportistas y rasgos de la personalidad. Incluir estas variables hubiese permitido describir de manera más global los resultados obtenidos. En próximas investigaciones es necesario cumplir con un análisis más detallado de la problemática del bullying en las escuelas deportivas cubanas. Además, los propios resultados del estudio sugieren la necesidad de utilizar dispositivos metodológicos longitudinales que nos permitan explorar con mayor precisión la relación entre variables (por ejemplo, las estrategias de regulación emocional) que pueden explicar la dinámica del bullying.

\section{CONCLUSIONES}

Los resultados del estudio proporcionan evidencia relacionada con los episodios de bullying en las escuelas deportivas cubanas, haciendo hincapié en el papel del género, las categorías deportivas (escolares y juveniles) y los deportes. Las conductas de bullying en las escuelas deportivas es un tema importante que merece mayor atención, especialmente en las cubanas donde los estudiantes están albergados, por lo que se requieren investigación y estrategias colaborativas que promuevan la prevención e intervención temprana en relación al bullying, incluyendo una base amplia de agentes socializadores como, por ejemplo, entrenadores, pares de entrenamiento y familiares. Todo esto puede contribuir a mejorar sustancialmente las relaciones interpersonales al interior de las escuelas y potenciar los resultados deportivos.

Conflictos de intereses: Ninguno. 


\section{REFERENCIAS BIBLIOGRÁFICAS}

Adler, A. L. (2014). An Examination into Bullying in the Adolescent Sport Context [Tesis doctoral]. Universidad de Queen. https://qspace.library.queensu.ca/bitstream/handle/1974/12 496/Adler_Adler_L_201409_MSc.pdf;jsessionid=712E01CA846DF88A613A335B08F3CA4 9? sequence=1

Ávila, J., Osorio, L., Cuello, K., Cogollo, N., \& Causado, K. (2010). Conducta bullying y su relación con la edad, género y nivel de formación en adolescentes. Psicogente, 13(23), 13-26. http://revistas.unisimon.edu.co/index.php/psicogente/article/view/1811

Bachand, C. R. (2017). Bullying in sports: The definition depends on who you ask. Sports Studies and Sports Psychology, 9, 1-14. https://thesportjournal.org/article/bullyingin-sports-the-definition-depends-on-whoyou-ask/

Björkqvist, K. (2018). Gender differences in aggression. Current Opinion in Psychology, 19, 3942. https://doi.org/10.1016/j.copsyc.2017.03.030

Brunstein, A., Sourander, A., \& Elonheimo, H. (2015). Bullying by peers in childhood and effects on psychopathology, suicidality, and criminality in adulthood. The Lancet Psychiatry, 2(10), 930-941. https://doi.org/10.1016/\$22150366(15)00223-0

Bullying sin Fronteras (2018). Bullying sin fronteras: Estadísticas de Bullying en América Latina. Primer Estudio Internacional. https://bullyingsinfronteras.blogspot.com/2018/02/estadisticas-debullying-en-america.html

Chai, L., Xue, J., \& Han, Z. (2020). School bullying victimization and self-rated health and life satisfaction: The mediating effect of relationships with parents, teachers, and peers. Children and Youth Services Review, 717, 105281. https://doi.org/10.1016/j.childyouth.2020.105 281
Domínguez-Alonso, J., López-Castedo, A., \& NietoCampos, B. (2019). Violencia escolar: diferencias de género en estudiantes de secundaria. Revista Complutense de Educación, $30(4)$,

1031-1044. https://doi.org/10.5209/rced.59997

Estrada, M. A., \& Jaik, A. (2011). Cuestionario para la exploración del bullying. Visión Educativa IUNAES, 5(11), 45-49. https://dialnet.unirioja.es/servlet/articulo?codigo $=4034505$

Evans, B., Adler, A., MacDonald, D., \& Côté, J. (2016). Bullying Victimization and Perpetration among Adolescent Sport Teammates. Pediatric Exercise Science, 28(2), 296-303. https://doi.org/10.1123/pes.2015-0088

Garaigordobil, M., \& Martínez-Valderrey, V. (2018). Technological resources to prevent cyberbullying during adolescence: the cyberprogram 2.0 program and the cooperative cybereduca 2.0 videogame. Frontiers in $\begin{array}{lll}\text { psychology, } & 9, & 745 .\end{array}$ https://doi.org/10.3389/fpsyg.2018.00745

Holbrook, H. M., Voller, F., Castellini, G., Silvestri, C., Ricca, V., Cassioli, E., ... Hudziak, J. J. (2020). Sport participation moderates association between bullying and depressive symptoms in Italian adolescents. Journal of Affective Disorders, 271, 33-38. https://doi.org/10.1016/j.jad.2020.03.142

Klomek, A. B., Sourander, A., \& Elonheimo, H. (2015). Bullying by peers in childhood and effects on psychopathology, suicidality, and criminality in adulthood. The Lancet Psychiatry, 2(10), 930-941. https://doi.org/10.1016/S22150366(15)00223-0

Machimbarrena, J. M., \& Garaigordobil, M. (2018). Bullying y cyberbullying: diferencias en función del sexo en estudiantes de quinto y sexto curso de educación primaria. Suma Psicológica, 25(2), 102-112. https://doi.org/10.14349/sumapsi.2018.v25.n2.2 
Malamut, S. T., van den Berg, Y. H., Lansu, T. A., \& Cillessen, A. H. (2020). Dyadic nominations of bullying: Comparing types of bullies and their victims. Aggressive behavior, 46(3), 232-243. https://doi.org/10.1002/ab.21884

Morcillo, C., Ramos-Olazagasti, M. A., Blanco, C., Sala, R., Canino, G., Bird, H., \& Duarte, C. S. (2014). Socio-Cultural Context and Bulling Others in Childhood. Journal of Child and Family Studies, 24, 2247-2249. https://doi.org/10.1007/s10826-014-0026-1

Nery, M., Neto, C., Rosado, A., \& Smith, P. K. (2018). Bullying in youth sport training: A nationwide exploratory and descriptive research in Portugal. European Journal of Developmental Psychology, 16(4), 447-463. https://doi.org/10.1080/17405629.2018.14474 59

ONG. (2019). Estadísticas mundiales de bullying 2017/2018. Primer trabajo oficial en el mundo contra el bullying. https://bullyingsinfronteras.blogspot.com/2018/10/estadisticasmundiales-de-bullying_29.html

Pepler, D. J., Craig, W. M., Connolly, J. A., Yuile, A., McMaster, L., \& Jiang, D. (2006). A developmental perspective on bullying. Aggressive Behavior, 32(4), 376-384. https://doi.org/10.1002/ab.20136

Rech, R. R., Halpern, R., Tedesco, A., \& Santos, D. F. (2013). Prevalence and characteristics of victims and perpetrators of bullying. Jornal de Pediatria, 89(2), 164-170. https://doi.org/10.1016/j.jpedp.2012.09.004
Ruiz, Y. (2018). Bullying en las escuelas. iAhora! http://www.ahora.cu/es/opinion/668-

bullying-en-las-escuelas

Silva, D., Tavares, E., Silva, E., Duarte, J., Cabral, L., y Martins, C. (2017). Vítimas e agressores: manifestações de bullying em alunos do $6^{\circ}$ ao $9^{\circ}$ ano de escolaridade. Revista Portuguesa de Enfermagem de Saúde Mental, (spe5), 57-62. http://dx.doi.org/10.19131/rpesm.0168

Sanmartín, J. (2007). ¿Qué es la violencia? Una aproximación al concepto y a la clasificación de la violencia. Daimon. Revista de Filosofía (42), 9-21. https://revistas.um.es/daimon/article/view/95881

Smokowski, P. R., \& Evans, C. B. (2019). Bullying and Victimization across the Lifespan: Playground Politics and Power. Springer.

Stirling, A. E., Bridges, E. J., Cruz, E. L., \& Mountjoy, M. L. (2011). Canadian Academy of Sport and Exercise Medicine position paper: Abuse, harassment, and bullying in sport. Clinical journal of sport medicine, 27(5), 385-391. http://dx.doi.org/10.1097 JSM.0b013e31820f9248

Vveinhardt, J., \& Fominiene, V. B. (2020). Gender and age variables of bullying in organized sport: Is bullying "grown out of"? Journal of Human Sport and Exercise, 15(4), 747-761. https://doi.org/10.14198/jhse.2020.154.03

\section{Dirección para correspondencia}

Mg. Osvaldo Hernández González

Universidad de Talca

Talca, Chile

ORCID: https://orcid.org/0000-0002-1319-6167

Contacto:

osvaldo.hernandez@utalca.cl

Recibido: 19-11-2020

Aceptado: 29-04-2021 